\title{
Pre-treatment expectations of patients with spinal metastases: what do we know and what can we learn from other disciplines? A systematic review of qualitative studies
}

R. Gal ${ }^{1 *}$ (D, D. Oostinga ${ }^{2}$, H. Wessels ${ }^{3}$, J. J. Verlaan², R. Charest-Morin ${ }^{4}$, C. G. Fisher ${ }^{4}$, H. M. Verkooijen ${ }^{1}$ and A. L. Versteeg ${ }^{1}$

\begin{abstract}
Background: Little is known about treatment expectations of patients with spinal metastases undergoing radiotherapy and/or surgery. Assuming that patients with spinal metastases share characteristics with patients who had spinal surgery for non-cancer related conditions and with advanced cancer patients, we performed a systematic review to summarize the literature on patient expectations regarding treatment outcomes of spinal surgery and advanced cancer care.

Methods: A comprehensive search was performed in MEDLINE, EMBASE and PsycINFO for studies between 2000 and sep-2019. Studies including adult patients (> 18 years), undergoing spinal surgery or receiving advanced cancer care, investigating patients' pre-treatment expectations regarding treatment outcomes were included. Two independent reviewers screened titles, abstracts and full-texts, extracted data and assessed methodological quality.

Results: The search identified 7343 articles, of which 92 were selected for full-text review. For this review, 31 articles were included. Patients undergoing spinal surgery had overly optimistic expectations regarding pain and symptom relief, they underestimated the probability of functional disability, and overestimated the probability of (complete) recovery and return to work. Studies highlighted that patients feel not adequately prepared for surgery in terms of post-treatment expectations. Similarly, advanced cancer patients receiving palliative treatment often had overly optimistic expectations regarding their survival probability and cure rates.

Conclusions: Patients tend to have overly optimistic expectations regarding pain and symptom relief, recovery and prognosis following spinal surgery or advanced cancer care. Pretreatment consultation about the expected pain and symptom relief, recovery and prognosis may improve understanding of prognosis, and promote and manage expectations, which, in turn, may lead to better perceived outcomes.
\end{abstract}

Trial registration: PROSPERO registration number: CRD42020145151.

Keywords: Spinal metastases, Treatment outcomes, Patient expectations, Spine surgery, Advanced cancer

\footnotetext{
* Correspondence: R.Gal@umcutrecht.nl

${ }^{1}$ Division of Imaging and Cancer, University Medical Center Utrecht, University of Utrecht, Heideberglaan 100, 3584, CG, Utrecht, the Netherlands

Full list of author information is available at the end of the article
}

(c) The Author(s). 2020 Open Access This article is licensed under a Creative Commons Attribution 4.0 International License, which permits use, sharing, adaptation, distribution and reproduction in any medium or format, as long as you give appropriate credit to the original author(s) and the source, provide a link to the Creative Commons licence, and indicate if changes were made. The images or other third party material in this article are included in the article's Creative Commons licence, unless indicated otherwise in a credit line to the material. If material is not included in the article's Creative Commons licence and your intended use is not permitted by statutory regulation or exceeds the permitted use, you will need to obtain permission directly from the copyright holder. To view a copy of this licence, visit http://creativecommons.org/licenses/by/4.0/. The Creative Commons Public Domain Dedication waiver (http://creativecommons.org/publicdomain/zero/1.0/) applies to the data made available in this article, unless otherwise stated in a credit line to the data. 


\section{Background}

Advances in the detection and treatment of cancer have increased survival rates over the last decades. With improved survival rates, more patients will develop spinal metastases, which can lead to devastating consequences including progressive and unremitting pain, spinal instability and metastatic epidural spinal cord compression (MESCC) [1-3]. Spinal metastases may therefore significantly impair the patients' health related quality of life (HRQOL). The treatment goal for patients with spinal metastases is to enhance HRQOL by suppressing symptoms for their remaining time. Radiotherapy has a central role in palliative treatment of uncomplicated painful spinal metastases, and aims to relieve pain and locally control the tumor. However, surgery may be required in case of mechanical pain, pathological fracture and/or neurological deficit caused by MESCC, with or without adjuvant radiotherapy [4].

A patient's HRQOL is a subjective and multidimensional construct and hence influenced by satisfaction with current health status, usually encompassing physical, emotional, and social functioning [5]. Pre-treatment expectations have been shown to play an important role in post-treatment quality of life [6]. When pre-treatment expectations are met after treatment, patients are more likely to be satisfied and perceive their post-treatment health status as more favorable, resulting in a higher quality of life [7-9]. When expectations are not met after treatment, patients will be less satisfied and hence, quality of life will be lower $[6,9]$.

Little is known about expectations of patients with spinal metastases undergoing radiotherapy and/or surgery. Assuming that patients with spinal metastases share characteristics with patients who had spinal surgery for non-cancer related conditions and with advanced cancer patients, we performed a metaaggregation to synthesize findings of published qualitative studies to explore patient expectations regarding treatment outcomes following spinal surgery and patient expectations regarding treatment outcomes in advanced cancer care. The objectives were to study patient expectations after treatment and to draw parallels with the metastatic spine population.

\section{Methods}

We followed the Preferred Reporting Items for Systematic Reviews and Meta-Analyses (PRISMA) statement [10]. The protocol was prospectively registered in the PROSPERO database (CRD42020145151).

\section{Search strategy and selection criteria}

Comprehensive searches were performed in the MEDL INE, EMBASE and PsycINFO databases. The search strategy was developed in close consultation with a university librarian. The search strategies can be found in Additional file 1. The search was restricted to articles in English or Dutch and published between 2000 and September 2019. Quantitative and qualitative studies that gave understanding of patients' pre-treatment expectations regarding treatment outcomes were included. Studies were eligible when they included adult patients (>18 years old) undergoing spinal surgery or with advanced cancer receiving palliative care. Two reviewers (AV and RG) independently screened titles and abstracts to identify articles requiring full-text review. The reference lists of included articles were searched for relevant articles. Next, full-text review was performed by two reviewers (DO and RG). If consensus was not reached, a third reviewer (AV) was consulted.

\section{Qualitative appraisal}

Quality of the included studies was independently assessed by two reviewers (DO and RG). Qualitative studies were assessed using The Critical Appraisal Skills Programme (CASP) tool for qualitative studies and cohort studies to assess study aims, methods, design, recruitment, data collection and analysis, researcherparticipants relationship, bias and confounding, ethics and reporting. The CASP tool for qualitative studies comprises 9 questions that are scored with 'yes', 'no' or 'can't tell' to explore whether study results are valid and relevant [11]. Question 10 was adapted to assess the relevance of the study results for this review. The CASP tool for cohort studies was modified; question 3 was removed since there is no exposure that is relevant for this systematic review, and questions $5 \mathrm{a}$ and $5 \mathrm{~b}$ were combined [12]. This resulted in 9 questions that are scored with 'yes', 'no' or 'can't tell'. Question 6 was only assessed when pre-treatment expectations and fulfillment of expectations were measured. Questions 7, 8 and 12 were used to assess the relevance of the study results for this review. Quality assessment was not used to exclude studies, but provided information about methodological rigor (i.e., appropriateness of the methods with regard to the study aims), credibility of findings (i.e., congruity between findings and supporting data), and robustness of included studies. This information was used as an indicator of the validity of the reported results and taken into account when interpreting the data.

\section{Data extraction}

Data from the included studies were extracted by two authors (DO and RG) using a predefined data extraction form that included information on the study characteristics (study aim, study population, methodology, phenome of interest). Relevant results (i.e., findings) as well as accompanying illustrations (i.e., quotations, statements or other textual extractions) supporting the 
findings were extracted from the included studies. A level of credibility (unequivocal, credible or not supported) was assigned to each finding [13]. Discrepancies between reviewers were discussed with a third author (AV).

\section{Data analysis}

Data synthesis of the extracted findings was done according to the meta-aggregation method developed by the Joanna Briggs Institute [14]. First, unequivocal and credible findings as extracted from the included studies were aggregated into categories based on similarity of outcomes. Next, these categories were combined based on similarity in concepts and outcomes, resulting in synthesized findings. Categories and synthesized findings were presented as statements, i.e., an overarching description that conveys the meaning of a set of categories or findings. Data synthesis was performed by one reviewer (RG). The data synthesis process was checked by, and discussed with a second reviewer (DO).

\section{Results}

\section{Screening and search outcomes}

The search strategy yielded 7343 articles. After removing duplicates, 5664 titles and abstracts were screened, resulting in 92 articles for full-text review (Fig. 1). In total, 31 articles met the inclusion criteria.

\section{Included studies}

The 31 studies were published between 2001 and 2019 (Table 1). Seventeen studies included patients undergoing spinal surgery and 14 studies included patients receiving advanced cancer care. In most studies, pretreatment expectations served as a determinant of a specific outcome such as post-operative pain, posttreatment fulfillment of expectations or satisfaction $[6,15,17,20-23,25-27,29,30,36-38,41,43]$. In other studies, the main objective was to gain insight into expectations [16, 18, 19, 24, 28, 31-35, 39, 40, 42, 44]. Methods to measure expectations included (semi-

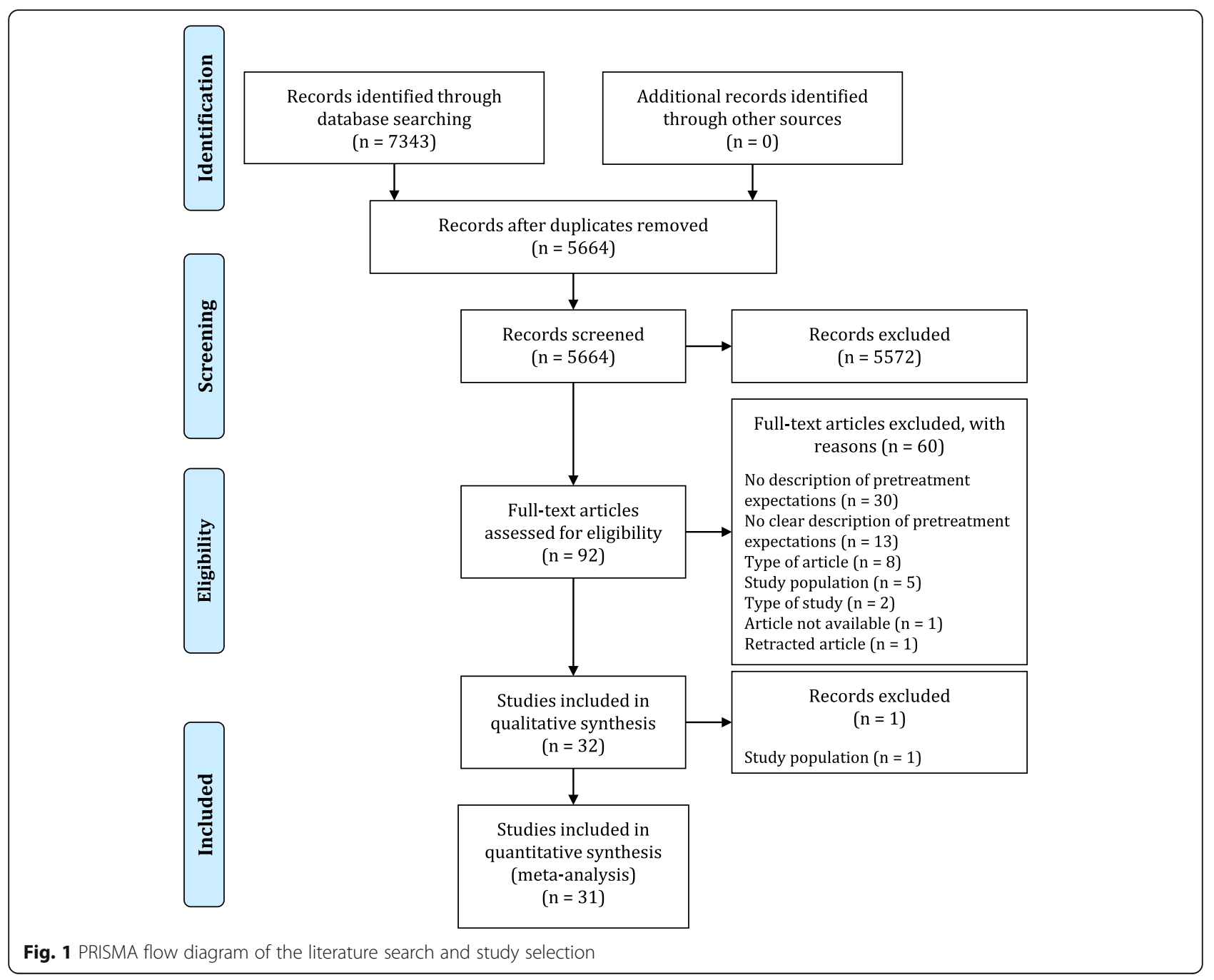


Table 1 Characteristics of the included studies

\begin{tabular}{|c|c|c|c|c|}
\hline $\begin{array}{l}\text { Author(s) } \\
\text { and year of } \\
\text { publication }\end{array}$ & Phenoma of interest & Study population & $\begin{array}{l}\text { Mean age } \\
\text { in years } \\
\text { Sex, } n(\%) \\
\text { female }\end{array}$ & $\begin{array}{l}\text { Method of data } \\
\text { collection } \\
\text { (expectations) } \\
\text { Timing of } \\
\text { measurement } \\
\end{array}$ \\
\hline \multicolumn{5}{|c|}{ Patients undergoing spinal surgery } \\
\hline $\begin{array}{l}\text { Accardi-Ravid } \\
\text { et al. (2019) [15] }\end{array}$ & $\begin{array}{l}\text { Preoperative and postoperative } \\
\text { experiences of spine surgery } \\
\text { including perioperative expectations, } \\
\text { emotional experiences, long-term } \\
\text { recovery, postoperative outcomes, } \\
\text { interest in perioperative psychosocial } \\
\text { interventions, and potential barriers } \\
\text { and facilitators to participating in an } \\
\text { intervention }\end{array}$ & 14 patients who had spine surgery & $\begin{array}{l}57.3(\text { SD 15.7) } \\
6(42.9)\end{array}$ & $\begin{array}{l}\text { Semi-structured } \\
\text { interviews } \\
2-12 \text { mo post-operatively }\end{array}$ \\
\hline $\begin{array}{l}\text { Lattig et al. } \\
\text { (2013) [16] }\end{array}$ & $\begin{array}{l}\text { Preoperative expectations of the } \\
\text { short-term results in relation to pain, } \\
\text { pain medication usage, sensory and } \\
\text { motor function, and the ability to } \\
\text { work, do household activities, and } \\
\text { participate in sports } \\
\text { Patient-surgeon discrepancies in } \\
\text { expectations }\end{array}$ & $\begin{array}{l}241 \text { patients (15-90y) undergoing } \\
\text { spine surgery }\end{array}$ & $\begin{array}{l}62(15) \\
133(59)\end{array}$ & $\begin{array}{l}\text { Survey } \\
\text { After preoperative } \\
\text { consultation }\end{array}$ \\
\hline $\begin{array}{l}\text { Licina et al. } \\
\text { (2012) [17] }\end{array}$ & $\begin{array}{l}\text { Expectations of the surgical } \\
\text { treatment (level of back and leg } \\
\text { pain, and disability), and satisfaction } \\
\text { with postoperative results }\end{array}$ & $\begin{array}{l}145 \text { patients scheduled for primary, } \\
\text { single-level surgery for degenerative } \\
\text { lumbar spine conditions }\end{array}$ & $\begin{array}{l}54(15) \\
54(37)\end{array}$ & $\begin{array}{l}\text { Survey } \\
\text { Preoperatively | } 6 \mathrm{w} / 6 \\
\text { mo post-operatively }\end{array}$ \\
\hline $\begin{array}{l}\text { Mancuso et al. } \\
\text { (2014) [18] }\end{array}$ & $\begin{array}{l}\text { Long-term expectations of surgery as } \\
\text { measured with the } 20 \text {-item Hospital } \\
\text { for Special Surgery, and associations } \\
\text { with demographic, clinical, and psy } \\
\text { chological characteristics }\end{array}$ & $\begin{array}{l}150 \text { patients ( } \geq 18 y) \text { scheduled for } \\
\text { cervical spine surgery }\end{array}$ & $\begin{array}{l}55(13) \\
59(39)\end{array}$ & $\begin{array}{l}\text { Interview (about survey) } \\
\text { Preoperatively }\end{array}$ \\
\hline $\begin{array}{l}\text { Mancuso et al. } \\
\text { (2015) [19] }\end{array}$ & $\begin{array}{l}\text { Associations between expectations } \\
\text { as measured with the 20-item } \\
\text { Hospital for Special Surgery, Lumbar/ } \\
\text { Cervical Spine Surgery Expectations } \\
\text { Survey, and demographic, } \\
\text { psychological, and clinical } \\
\text { characteristics }\end{array}$ & $\begin{array}{l}420 \text { patients ( } \geq 18 y \text { ) scheduled for } \\
\text { lumbar spine surgery }\end{array}$ & $\begin{array}{l}55(15) \\
181(43)\end{array}$ & $\begin{array}{l}\text { Interview (about survey) } \\
\pm 7 \text { d post-operatively }\end{array}$ \\
\hline $\begin{array}{l}\text { Mancuso et al. } \\
(2016)[20]\end{array}$ & $\begin{array}{l}\text { Preoperatively stated expectations as } \\
\text { measured with the } 20 \text {-item Hospital } \\
\text { for Special Surgery, Lumbar/Cervical } \\
\text { Spine Surgery Expectations Survey } \\
\text { and fulfillment of expectations post- } \\
\text { operatively }\end{array}$ & $\begin{array}{l}\text { Patients ( } \geq 18 y) \text { scheduled for lumbar } \\
(n=366) \text { or cervical }(n=133) \text { spine } \\
\text { surgery }\end{array}$ & $\begin{array}{l}\text { Lumbar: } \\
55 \text { (SD 15) } \\
157 \text { (43) } \\
\text { Cervical: } \\
54 \text { (SD 13) } \\
51 \text { (133) }\end{array}$ & $\begin{array}{l}\text { Survey: } \pm 7 \text { d } \\
\text { preoperatively } \\
\text { Interview (telephone): } \\
2 \text { y post-operatively }\end{array}$ \\
\hline $\begin{array}{l}\text { Mancuso et al. } \\
\text { (2017) [21] }\end{array}$ & $\begin{array}{l}\text { Patient and clinical characteristics, } \\
\text { including the } 20 \text {-item Hospital for } \\
\text { Special Surgery, Lumbar/Cervical } \\
\text { Spine Surgery Expectations Survey, } \\
\text { and pain improvement post- } \\
\text { operatively }\end{array}$ & $\begin{array}{l}422 \text { patients ( } \geq 18 y) \text { scheduled } \\
\text { for lumbar spine surgery }\end{array}$ & $\begin{array}{l}56(15) \\
190(45)\end{array}$ & $\begin{array}{l}\text { Interview (structured): } \\
\pm 7 \text { d preoperatively } \\
\text { Interview (telephone): } \\
2 \text { y post- operatively }\end{array}$ \\
\hline $\begin{array}{l}\text { Mannion et al. } \\
\text { (2009) [22] }\end{array}$ & $\begin{array}{l}\text { Preoperative expectations as } \\
\text { measured with a modified version of } \\
\text { the "expectations scale" of the North } \\
\text { American Spine Society (NASS) } \\
\text { Lumbar Spine Questionnaire, } \\
\text { changes in symptoms, and } \\
\text { expectations being fulfilled }\end{array}$ & $\begin{array}{l}100 \text { patients (>45y) with lumbar } \\
\text { herniated disc or spinal stenosis, } \\
\text { indication for decompression } \\
\text { surgery without fusion }\end{array}$ & $\begin{array}{l}65(\mathrm{SD} 11) \\
33(33)\end{array}$ & $\begin{array}{l}\text { Survey } \\
\text { Preoperatively | } 2 \text { mo/12 } \\
\text { mo post-operatively }\end{array}$ \\
\hline $\begin{array}{l}\text { McGregor et al. } \\
\text { (2013) [23] }\end{array}$ & $\begin{array}{l}\text { Preoperative expectations (e.g. state } \\
\text { of health and their levels of back } \\
\text { and leg pain) and importance of } \\
\text { achieving this level of recovery, }\end{array}$ & $\begin{array}{l}316 \text { patients scheduled for lumbar } \\
\text { decompression or discectomy } \\
\text { because of lateral nerve root } \\
\text { compression or lumbar disc prolapse }\end{array}$ & $\begin{array}{l}\text { range } 53-55 \\
170(54)\end{array}$ & $\begin{array}{l}\text { Survey } \\
\text { Preoperatively | } 6 \mathrm{w} / 6 \\
\text { mo/12 mo post- } \\
\text { operatively }\end{array}$ \\
\hline
\end{tabular}


Table 1 Characteristics of the included studies (Continued)

\begin{tabular}{|c|c|c|c|c|}
\hline $\begin{array}{l}\text { Author(s) } \\
\text { and year of } \\
\text { publication }\end{array}$ & Phenoma of interest & Study population & $\begin{array}{l}\text { Mean age } \\
\text { in years } \\
\text { Sex, } n(\%) \\
\text { female }\end{array}$ & $\begin{array}{l}\text { Method of data } \\
\text { collection } \\
\text { (expectations) } \\
\text { Timing of } \\
\text { measurement } \\
\end{array}$ \\
\hline & $\begin{array}{l}\text { longer term outcome of surgery } \\
\text { (in terms of pain and QoL) }\end{array}$ & & & \\
\hline $\begin{array}{l}\text { Rehman et al. } \\
\text { (2019) [24] }\end{array}$ & $\begin{array}{l}\text { Preoperative expectations and the } \\
\text { spine surgeon's perspectives } \\
\text { regarding treatment understanding, } \\
\text { postoperative outcomes and } \\
\text { information required for informed } \\
\text { decision-making }\end{array}$ & $\begin{array}{l}12 \text { patients ( } \geq 18 y \text { ) with sciatica, } \\
\text { scheduled for surgical } \\
\text { decompression } \\
6 \text { surgeons }\end{array}$ & $\begin{array}{l}\text { Patients: } \\
\text { median } 48 \\
\text { (range 24-74) } \\
5 \text { (43) } \\
\text { Surgeons: } \\
\text { median } 50 \\
\text { (range 45-68)- }\end{array}$ & $\begin{array}{l}\text { Semi-structured } \\
\text { interviews } \\
3-4 \mathrm{w} \text { after consultation, } \\
\text { but preoperatively }\end{array}$ \\
\hline $\begin{array}{l}\text { Rönnberg et al. } \\
\text { (2007) [25] }\end{array}$ & $\begin{array}{l}\text { Relationships between baseline } \\
\text { characteristics and expectations of } \\
\text { surgical results (leg pain, back pain, } \\
\text { sensibility, return to work), and } \\
\text { satisfaction with provided care and } \\
\text { given information }\end{array}$ & $\begin{array}{l}148 \text { patients who had undergone } \\
\text { surgery for a one-level disc } \\
\text { herniation on the } L 4-L 5 \text { or } \\
\text { L5-S1 level }\end{array}$ & $\begin{array}{l}40 \text { (range 18-66) } \\
68(46)\end{array}$ & $\begin{array}{l}\text { Survey } \\
\text { Preoperatively | } 2 \text { y post- } \\
\text { operatively }\end{array}$ \\
\hline $\begin{array}{l}\text { Saban and } \\
\text { Penckofer } \\
\text { (2007) [6] }\end{array}$ & $\begin{array}{l}\text { Relationship between preoperative } \\
\text { expectations of QoL, and } \\
\text { postoperative perceived } \mathrm{QOL} \text { and } \\
\text { level of satisfaction and optimism }\end{array}$ & $\begin{array}{l}57 \text { patients ( } \geq 18 y) \text { undergoing } \\
\text { elective lumbar surgery for the } \\
\text { first time for degenerative } \\
\text { changes, herniated disks, or } \\
\text { both }\end{array}$ & $\begin{array}{l}53.4(\mathrm{SD} 13.6) \\
30(52.6)\end{array}$ & $\begin{array}{l}\text { Survey } \\
2-14 \text { d preoperatively } \\
3 \text { m post-operatively }\end{array}$ \\
\hline $\begin{array}{l}\text { Soroceanu et al. } \\
\text { (2012) [26] }\end{array}$ & $\begin{array}{l}\text { Relationship between expectations } \\
\text { as measured with the } \\
\text { Musculoskeletal Outcomes Data } \\
\text { Evaluation and Management } \\
\text { System's (MODEMS) expectations } \\
\text { survey, and outcomes in the cervical } \\
\text { versus the lumbar spine population }\end{array}$ & $\begin{array}{l}402 \text { patients undergoing lumbar } \\
\text { or cervical spine surgery }\end{array}$ & $\begin{array}{l}52.9(15.2) \\
226(56.3)\end{array}$ & $\begin{array}{l}\text { Survey } \\
\text { Preoperatively | 6-12 w } \\
\text { post-operatively }\end{array}$ \\
\hline $\begin{array}{l}\text { Toyone et al. } \\
\text { (2005) [27] }\end{array}$ & $\begin{array}{l}\text { Patient expectations of spine surgery } \\
\text { including relief of leg pain, leg } \\
\text { numbness and low back pain, and } \\
\text { limitations in walking ability and } \\
\text { activity of daily living, and the level } \\
\text { of fulfillment of those expectations }\end{array}$ & $\begin{array}{l}\text { Patients undergoing lumbar disc } \\
\text { herniation }(n=49) \text { or lumbar } \\
\text { spinal stenosis }(n=49)\end{array}$ & $\begin{array}{l}\text { Disc herniation: } \\
36 \\
15(31) \\
\text { Spinal stenosis: } \\
67 \\
22(45)\end{array}$ & $\begin{array}{l}\text { Survey } \\
\text { Preoperatively | } 2 \text { y } \\
\text { post-operatively }\end{array}$ \\
\hline $\begin{array}{l}\text { van der Horst } \\
\text { et al. (2019) [28] }\end{array}$ & $\begin{array}{l}\text { Pre-operative expectations and } \\
\text { perceptions, and post-operative } \\
\text { experiences (e.g. limitations in daily } \\
\text { functioning due to their back pain, } \\
\text { other health complaints) }\end{array}$ & $\begin{array}{l}12 \text { patients ( } \geq 18 \mathrm{y}) \text { who had } \\
\text { spinal fusion in last } 6 \mathrm{mo}\end{array}$ & $-\overline{6}(50)$ & $\begin{array}{l}\text { Survey } \\
\text { 0-9 mo post-operatively }\end{array}$ \\
\hline $\begin{array}{l}\text { Yee et al. } \\
\text { (2008) [29] }\end{array}$ & $\begin{array}{l}\text { Expectations of surgery (regarding } \\
\text { relief of back and leg pain, relief of } \\
\text { numbness/weakness/instability, their } \\
\text { ability to sleep, recreational and daily } \\
\text { activities, and return to work), and its } \\
\text { association with patient factors, } \\
\text { baseline preoperative functional } \\
\text { outcome scores and patient- } \\
\text { reported improvements in functional } \\
\text { outcome after surgery }\end{array}$ & $\begin{array}{l}143 \text { patients undergoing } \\
\text { decompression and/or } \\
\text { spondylodesis (spinal fusion) }\end{array}$ & $\begin{array}{l}52 \text { (range 18-84) } \\
50 \%\end{array}$ & $\begin{array}{l}\text { Survey } \\
\text { Preoperatively | } 6 \mathrm{w} / 3 \\
\text { mo/6 mo/12 mo post- } \\
\text { operatively }\end{array}$ \\
\hline $\begin{array}{l}\text { Yoo et al. } \\
(2019)[30]\end{array}$ & $\begin{array}{l}\text { Preoperative expectations and } \\
\text { postoperative outcomes, and the } \\
\text { effect on postoperative satisfaction }\end{array}$ & $\begin{array}{l}101 \text { patients (>18y) undergoing 1- } \\
\text { or 2-level minimally invasive spinal } \\
\text { lumbar fusion surgery for } \\
\text { degenerative pathology }\end{array}$ & $\begin{array}{l}57 \\
43(42.6)\end{array}$ & $\begin{array}{l}\text { Survey } \\
\text { Preoperatively | } 6 \text { mo } \\
\text { post-operatively }\end{array}$ \\
\hline \multicolumn{5}{|c|}{ Patients receiving advanced cancer care } \\
\hline $\begin{array}{l}\text { Bergerot et al. } \\
(2019)[31]\end{array}$ & $\begin{array}{l}\text { Association between expectations } \\
\text { of cure and QoL, anxiety and } \\
\text { depression }\end{array}$ & $\begin{array}{l}60 \text { patients with metastatic renal cell } \\
\text { carcinoma, urothelial carcinoma or } \\
\text { prostate cancer }\end{array}$ & $\begin{array}{l}65.1 \text { (SD 13.1; range } \\
31-91) \\
19(31.7)\end{array}$ & $\begin{array}{l}\text { Survey } \\
\text { Prior to immunotherapy } \\
\text { and before counseling } \\
\text { from their oncologist }\end{array}$ \\
\hline
\end{tabular}


Table 1 Characteristics of the included studies (Continued)

\begin{tabular}{|c|c|c|c|c|}
\hline $\begin{array}{l}\text { Author(s) } \\
\text { and year of } \\
\text { publication }\end{array}$ & Phenoma of interest & Study population & $\begin{array}{l}\text { Mean age } \\
\text { in years } \\
\text { Sex, } n(\%) \\
\text { female }\end{array}$ & $\begin{array}{l}\text { Method of data } \\
\text { collection } \\
\text { (expectations) } \\
\text { Timing of } \\
\text { measurement }\end{array}$ \\
\hline $\begin{array}{l}\text { Chen et al. } \\
\text { (2013) [32] }\end{array}$ & $\begin{array}{l}\text { Expectations about the goals of, and } \\
\text { likelihood of cure from radiotherapy }\end{array}$ & $\begin{array}{l}384 \text { patients with incurable lung } \\
\text { cancer (stage IV or IIIB) }\end{array}$ & $\begin{array}{l}\text { median: } 63 \\
154(40)\end{array}$ & $\begin{array}{l}\text { Telephone survey } \\
4-7 \text { mo post-diagnosis }\end{array}$ \\
\hline $\begin{array}{l}\text { Chow et al. } \\
\text { (2001) [33] }\end{array}$ & $\begin{array}{l}\text { Illness understanding and } \\
\text { expectations of palliative } \\
\text { radiotherapy }\end{array}$ & $\begin{array}{l}60 \text { patients with advanced cancer, } \\
\text { referred for palliative radiotherapy }\end{array}$ & $\begin{array}{l}\text { median } 68 \\
\text { (range 46-90) } \\
30(50)\end{array}$ & $\begin{array}{l}\text { Survey } \\
\text { Pre-consultation }\end{array}$ \\
\hline $\begin{array}{l}\text { Chow et al. } \\
\text { (2007) [34] }\end{array}$ & $\begin{array}{l}\text { Expected level of pain reduction, and } \\
\text { influence of bone pain and having } \\
\text { undergone the treatment on this } \\
\text { expectation }\end{array}$ & $\begin{array}{l}217 \text { patients }(\geq 18 \mathrm{y}) \text { with bone } \\
\text { metastases, treated with palliative RT }\end{array}$ & $\begin{array}{l}\text { median: 66 } \\
\text { (range 28-88) } \\
87(40)\end{array}$ & $\begin{array}{l}\text { Interview } \\
\text { Pre-radiation | } 2 \text { mo post- } \\
\text { radiation }\end{array}$ \\
\hline $\begin{array}{l}\text { Craft et al. } \\
\text { (2005) [35] }\end{array}$ & $\begin{array}{l}\text { Understanding of the intent of } \\
\text { their treatment (to monitor illness, } \\
\text { improve } \mathrm{Q} \mathrm{L} \text {, control illness or cure } \\
\text { illness) and that their illness was life- } \\
\text { threatening, and sources of } \\
\text { information }\end{array}$ & $\begin{array}{l}163 \text { patients (>18y) with advanced } \\
\text { cancer }\end{array}$ & $-89(55)$ & $\begin{array}{l}\text { Survey } \\
\text { Week } 1 \text { and } 12\end{array}$ \\
\hline $\begin{array}{l}\text { Doyle et al. } \\
\text { (2001) [36] }\end{array}$ & $\begin{array}{l}\text { Patient expectations and perceptions } \\
\text { of benefit }\end{array}$ & $\begin{array}{l}26 \text { patients with recurrent or } \\
\text { refractory advanced ovarian cancer, } \\
\text { undergoing } 2 \text { nd or } 3 \text { nd line } \\
\text { chemotherapy for }\end{array}$ & $\begin{array}{l}\text { median: } 55 \\
26(100)\end{array}$ & $\begin{array}{l}\text { Surgery } \\
\text { Before chemotherapy }\end{array}$ \\
\hline $\begin{array}{l}\text { Friedlander } \\
\text { et al. (2014) [37] }\end{array}$ & $\begin{array}{l}\text { Symptom burden, and expected and } \\
\text { perceived benefits of chemotherapy }\end{array}$ & $\begin{array}{l}126 \text { patients with platinum resistant } \\
\text { ovarian cancer and a life expectancy } \\
\text { of }>3 \text { mo, scheduled for } \\
\text { chemotherapy }\end{array}$ & $\begin{array}{l}62 \text { (range 30-89) } \\
126(100)\end{array}$ & $\begin{array}{l}\text { Survey } \\
<2 \text { w before } \\
\text { chemotherapy | before } \\
\text { each cycle | } 4 \text { w after } 4 \text { th } \\
\text { cycle }\end{array}$ \\
\hline $\begin{array}{l}\text { Gramling et al. } \\
\text { (2016) [38] }\end{array}$ & $\begin{array}{l}\text { Association between talking about } \\
\text { expectations for length of life during } \\
\text { inpatient palliative care consultations } \\
\text { and rates of hospice enrollment }\end{array}$ & $\begin{array}{l}101 \text { hospitalized patients (>21y) with } \\
\text { metastatic cancer, referred for } \\
\text { palliative care consultation }\end{array}$ & $50(43.5)$ & $\begin{array}{l}\text { Audio-recording of } \\
\text { the consultation }\end{array}$ \\
\hline $\begin{array}{l}\text { Mitera et al. } \\
\text { (2012) [39] }\end{array}$ & $\begin{array}{l}\text { Illness understanding and } \\
\text { expectations of palliative } \\
\text { radiotherapy }\end{array}$ & $\begin{array}{l}100 \text { patients with advanced cancer, } \\
\text { referred for a palliative radiotherapy } \\
\text { consultation }\end{array}$ & $\begin{array}{l}66.2(11.3) \\
44(44)\end{array}$ & $\begin{array}{l}\text { Survey } \\
\text { Pre-consultation / } \\
\text { Post-consultation }\end{array}$ \\
\hline $\begin{array}{l}\text { Nowicki et al. } \\
\text { (2015) [40] }\end{array}$ & $\begin{array}{l}\text { Understanding and expectations of } \\
\text { treatment, and socio-demographic } \\
\text { factors }\end{array}$ & $\begin{array}{l}100 \text { patients with lung cancer and a } \\
\text { life expectancy of }>6 \text { mo, } \\
\text { undergoing palliative chemotherapy } \\
\text { and an }\end{array}$ & $\begin{array}{l}63.1 \text { (range } 40-80 \text { ) } \\
34(34)\end{array}$ & $\begin{array}{l}\text { Survey } \\
?\end{array}$ \\
\hline $\begin{array}{l}\text { Sjoquist et al. } \\
\text { (2013) [41] }\end{array}$ & $\begin{array}{l}\text { We explored associations among } \\
\text { expected improvement, hope and } \\
\text { indices of well-being, and perceived } \\
\text { symptom benefits of chemotherapy }\end{array}$ & $\begin{array}{l}126 \text { patients }(\geq 18 \mathrm{y}) \text { with recurrent } \\
\text { and progressive ovarian cancer and } \\
\text { a life expectancy of } \geq 3 \mathrm{mo}\end{array}$ & $\begin{array}{l}62.1(9.8) \\
126(100)\end{array}$ & $\begin{array}{l}\text { Survey } \\
\text { Prior to chemotherapy | } \\
\text { first four treatment cycles } \\
\text { | } 4 \text { w post-treatment }\end{array}$ \\
\hline $\begin{array}{l}\text { Sze et al. } \\
\text { (2006) [42] }\end{array}$ & $\begin{array}{l}\text { Factors important in decision making } \\
\text { for whole-brain radiation therapy for } \\
\text { patients and caregivers }\end{array}$ & $\begin{array}{l}20 \text { patients (or caregivers) with brain } \\
\text { metastases within the past } 2 \text { mo, } \\
\text { consideration of brain radiotherapy }\end{array}$ & $\begin{array}{l}\text { median: } 62 \\
\text { (range } 50-72) \\
5(25)\end{array}$ & $\begin{array}{l}\text { Open-ended, } \\
\text { semistructured interviews } \\
\text { Within } 2 \mathrm{w} \text { after } \\
\text { consultation }\end{array}$ \\
\hline $\begin{array}{l}\text { Visser et al. } \\
\text { (2018) [43] }\end{array}$ & $\begin{array}{l}\text { Satisfaction with therapy as } \\
\text { measured with the Cancer Therapy } \\
\text { Satisfaction Questionnaire (CTSQ), } \\
\text { and patient- and treatment-related } \\
\text { factors and patients' feelings about } \\
\text { adverse events }\end{array}$ & $\begin{array}{l}65 \text { patients with locally advanced or } \\
\text { metastatic stage IIIB/IV nonsquamous } \\
\text { NSCLC, undergoing chemotherapy }\end{array}$ & $\begin{array}{l}62.1(7.9) \\
50(50)\end{array}$ & $\begin{array}{l}\text { Survey } \\
\text { During 4th cycle } \\
\text { of chemotherapy }\end{array}$ \\
\hline $\begin{array}{l}\text { Weeks et al. } \\
\text { (2012) [44] }\end{array}$ & $\begin{array}{l}\text { Expectation that chemotherapy } \\
\text { might be curative and associated } \\
\text { clinical, sociodemographic, and } \\
\text { health-system factors, and physician } \\
\text { communication }\end{array}$ & $\begin{array}{l}\text { Patients with stage IV (i.e., } \\
\text { metastatic) lung }(n=710) \text { or } \\
\text { colorectal cancer }(n=483) \text {, } \\
\text { opted to receive chemotherapy }\end{array}$ & $\begin{array}{l}\text { Lung: } 476 \text { (67) } \\
\text { Colorectal: } 396 \text { (82) }\end{array}$ & $\begin{array}{l}\text { Structured interview } \\
4-7 \text { mo post-diagnosis }\end{array}$ \\
\hline
\end{tabular}


Table 2 Quality appraisal of studies

\begin{tabular}{|c|c|c|c|c|c|c|c|c|c|c|}
\hline \multicolumn{11}{|c|}{ Qualitative studies } \\
\hline & $\begin{array}{l}\text { 1. Clear } \\
\text { statement } \\
\text { of } \\
\text { research } \\
\text { aims? }\end{array}$ & $\begin{array}{l}\text { 2. Qualitative } \\
\text { methodology } \\
\text { appropriate? }\end{array}$ & $\begin{array}{l}\text { 3. Appropriate } \\
\text { research } \\
\text { design? }\end{array}$ & $\begin{array}{l}\text { 4. Appropriate } \\
\text { recruitment } \\
\text { strategy? }\end{array}$ & $\begin{array}{l}\text { 5. Data } \\
\text { collection in } \\
\text { a way that } \\
\text { addressed } \\
\text { research } \\
\text { issue? }\end{array}$ & $\begin{array}{l}\text { 6. Relationship } \\
\text { between } \\
\text { researcher and } \\
\text { participants } \\
\text { adequately } \\
\text { considered? }\end{array}$ & $\begin{array}{l}\text { 7. Ethical } \\
\text { issues } \\
\text { considered? }\end{array}$ & $\begin{array}{l}\text { 8. Sufficiently } \\
\text { rigorous data } \\
\text { analysis? }\end{array}$ & $\begin{array}{l}\text { 9. Clear } \\
\text { statement } \\
\text { of findings? }\end{array}$ & $\begin{array}{l}\text { 10. Results } \\
\text { valuable } \\
\text { for this } \\
\text { review? }\end{array}$ \\
\hline $\begin{array}{l}\text { Accardi- } \\
\text { Ravid et al. } \\
\text { (2019) [15] }\end{array}$ & Y & Y & Y & Y & Y & Y & N & Y & Y & Y \\
\hline $\begin{array}{l}\text { Rehman } \\
\text { et al. } \\
\text { (2019) [24] }\end{array}$ & Y & Y & Y & Y & Y & $N$ & Y & Y & Y & Y \\
\hline $\begin{array}{l}\text { Sze et al. } \\
\text { (2006) [42] }\end{array}$ & Y & Y & Y & Y & Y & $?$ & Y & Y & Y & Y \\
\hline $\begin{array}{l}\text { van der } \\
\text { Horst et al. } \\
\text { (2019) [28] }\end{array}$ & Y & Y & Y & Y & Y & N & Y & Y & Y & Y \\
\hline \multicolumn{11}{|c|}{ Cohort studies } \\
\hline & $\begin{array}{l}\text { 1. Clearly } \\
\text { focused } \\
\text { issue? }\end{array}$ & $\begin{array}{l}\text { 2. Acceptable } \\
\text { recruitment? }\end{array}$ & $\begin{array}{l}\text { 3. Outcome } \\
\text { accurately } \\
\text { measured } \\
\text { to minimise } \\
\text { bias? }\end{array}$ & $\begin{array}{l}\text { 4. All } \\
\text { confounding } \\
\text { factors } \\
\text { identified } \\
\text { and taken } \\
\text { into account? }\end{array}$ & $\begin{array}{l}\text { 5. Complete } \\
\text { follow-up? }\end{array}$ & $\begin{array}{l}\text { 6. Follow-up } \\
\text { long enough? }\end{array}$ & $\begin{array}{l}\text { 7. Do you } \\
\text { believe the } \\
\text { results? }\end{array}$ & $\begin{array}{l}\text { 8. Results } \\
\text { generalizable? }\end{array}$ & $\begin{array}{l}\text { 9. Results } \\
\text { fit with } \\
\text { other } \\
\text { available } \\
\text { evidence? }\end{array}$ & $\begin{array}{l}\text { 10. Results } \\
\text { valuable } \\
\text { for this } \\
\text { review? }\end{array}$ \\
\hline $\begin{array}{l}\text { Bergerot } \\
\text { et al. } \\
\text { (2019) [31] }\end{array}$ & Y & Y & Y & Y & NA & NA & Y & N & Y & Y \\
\hline $\begin{array}{l}\text { Chen et al. } \\
\text { (2013) [32] }\end{array}$ & Y & Y & Y & Y & $N$ & NA & Y & N & Y & Y \\
\hline $\begin{array}{l}\text { Chow et al. } \\
\text { (2001) [33] }\end{array}$ & Y & $?$ & Y & Y & NA & NA & Y & $\mathrm{N}$ & Y & Y \\
\hline $\begin{array}{l}\text { Chow et al. } \\
\text { (2001) [34] }\end{array}$ & Y & Y & Y & Y & $N$ & $N$ & Y & $\mathrm{N}$ & Y & Y \\
\hline $\begin{array}{l}\text { Craft et al. } \\
\text { (2005) [35] }\end{array}$ & Y & Y & Y & Y & Y & Y & Y & Y & Y & Y \\
\hline $\begin{array}{l}\text { Doyle et al. } \\
\text { (2001) [36] }\end{array}$ & Y & Y & Y & Y & Y & N & Y & N & Y & Y \\
\hline $\begin{array}{l}\text { Friedlander } \\
\text { et al. } \\
\text { (2014) [37] }\end{array}$ & Y & N & Y & Y & Y & Y & Y & Y & Y & Y \\
\hline $\begin{array}{l}\text { Gramling } \\
\text { et al. } \\
\text { (2016) [38] }\end{array}$ & Y & Y & N & $N$ & Y & NA & Y & $\mathrm{N}$ & Y & Y \\
\hline $\begin{array}{l}\text { Lattig et al. } \\
\text { (2013) [16] }\end{array}$ & Y & Y & Y & Y & Y & NA & Y & $?$ & Y & Y \\
\hline $\begin{array}{l}\text { Licina et al. } \\
\text { (2012) [17] }\end{array}$ & Y & Y & Y & Y & Y & Y & Y & Y & $?$ & Y \\
\hline $\begin{array}{l}\text { Mancuso } \\
\text { et al. } \\
\text { (2014) [18] }\end{array}$ & Y & Y & Y & $\mathrm{N}$ & Y & NA & Y & $\mathrm{N}$ & Y & Y \\
\hline $\begin{array}{l}\text { Mancuso } \\
\text { et al. } \\
\text { (2015) [19] }\end{array}$ & Y & Y & Y & $N$ & Y & Y & Y & $N$ & Y & Y \\
\hline $\begin{array}{l}\text { Mancuso } \\
\text { et al. } \\
\text { (2016) [20] }\end{array}$ & Y & Y & Y & Y & Y & Y & Y & N & Y & Y \\
\hline $\begin{array}{l}\text { Mancuso } \\
\text { et al. } \\
\text { (2017) [21] }\end{array}$ & Y & Y & Y & N & Y & Y & Y & N & Y & Y \\
\hline $\begin{array}{l}\text { Mannion } \\
\text { et al. } \\
\text { (2009) [22] }\end{array}$ & Y & $\mathrm{N}$ & Y & $N$ & Y & Y & Y & $?$ & Y & Y \\
\hline
\end{tabular}


Table 2 Quality appraisal of studies (Continued)

\begin{tabular}{|c|c|c|c|c|c|c|c|c|c|c|}
\hline $\begin{array}{l}\text { McGregor } \\
\text { et al. } \\
\text { (2013) [23] }\end{array}$ & $Y$ & $Y$ & Y & $Y$ & Y & $Y$ & $Y$ & $N$ & $Y$ & $Y$ \\
\hline $\begin{array}{l}\text { Mitera } \\
\text { et al. } \\
\text { (2012) [39] }\end{array}$ & Y & $?$ & $Y$ & $Y$ & Y & Y & $Y$ & $Y$ & $Y$ & Y \\
\hline $\begin{array}{l}\text { Nowicki } \\
\text { et al. } \\
\text { (2015) [40] }\end{array}$ & Y & Y & Y & Y & Y & NA & Y & Y & Y & $Y$ \\
\hline $\begin{array}{l}\text { Rönnberg } \\
\text { et al. } \\
\text { (2007) [25] }\end{array}$ & Y & Y & $Y$ & Y & Y & Y & Y & Y & $Y$ & $Y$ \\
\hline $\begin{array}{l}\text { Saban \& } \\
\text { Penckofer } \\
\text { (2007) [6] }\end{array}$ & Y & Y & Y & Y & $N$ & Y & Y & $N$ & Y & Y \\
\hline $\begin{array}{l}\text { Sjoquist } \\
\text { et al. } \\
\text { (2013) [41] }\end{array}$ & $Y$ & Y & Y & Y & Y & Y & Y & Y & Y & $Y$ \\
\hline $\begin{array}{l}\text { Soroceanu } \\
\text { et al. } \\
\text { (2012) [26] }\end{array}$ & Y & Y & Y & $Y$ & $?$ & Y & Y & $N$ & Y & $Y$ \\
\hline $\begin{array}{l}\text { Toyone } \\
\text { et al. } \\
\text { (2005) [27] }\end{array}$ & Y & $?$ & Y & $Y$ & Y & Y & Y & Y & Y & Y \\
\hline $\begin{array}{l}\text { Visser et al. } \\
\text { (2018) [43] }\end{array}$ & $Y$ & Y & Y & Y & $N$ & NA & Y & $N$ & Y & $Y$ \\
\hline $\begin{array}{l}\text { Weeks } \\
\text { et al. } \\
\text { (2012) [44] }\end{array}$ & Y & Y & Y & $Y$ & NA & NA & Y & $N$ & Y & $Y$ \\
\hline $\begin{array}{l}\text { Yee et al. } \\
\text { (2008) [29] }\end{array}$ & Y & Y & Y & $Y$ & Y & Y & Y & Y & Y & $Y$ \\
\hline $\begin{array}{l}\text { Yoo et al. } \\
\text { (2019) [30] }\end{array}$ & Y & Y & Y & Y & Y & Y & Y & $N$ & Y & $Y$ \\
\hline
\end{tabular}

$N$ no, NA not applicable, $Y$ yes; $=$ can't tell

structured) interviews, surveys and one study used audiorecordings of the consultation.

The quality appraisal showed that the overall quality of the included studies was high (Table 2). However, most studies scored "no" on the item assessing the generalizability of the results because these studies enrolled patients from one (specialized) center, had a small sample size, or experienced selective drop-out [6, 18-21, $23,26,30-34,36,38,43,44]$.

\section{Synthesized findings}

In total, 78 findings with 152 illustrations were extracted from the 31 included studies and assessed as credible or unequivocal. These were aggregated into 12 categories, which were combined to 3 synthesized findings.

Finding 1: the majority of patients expected improvement on several domains after treatment, but these expectations were often overly optimistic

Overall, patients scheduled for spinal surgery expected relief of their symptoms and improvement in physical functioning and undertaking daily activities after surgery (Table 3). However, many studies reported that patients had high and overly optimistic expectations of post- operative outcomes in terms of reduction in (back and leg) pain, symptom relief and better physical functioning $[6,16,17,22,24,25,28,31-33,35,36,39,40,42-44]$. For example, Mannion et al. concluded from their study on patients who had decompression spinal surgery that "the expectations declared before surgery had been overly optimistic in approximately 30\% to $40 \%$ patients for leg pain, back pain, walking capacity, independence, social function, and mental well-being, and in almost 50\% patients for general physical capacity at home and work (general function) and sport." [22]

Rehman et al. conducted interviews with surgeons and concluded the following: "More often than not, surgeons reported that patients were overly optimistic about surgery, expecting complete recovery, including their back pain: "... often patients think their symptoms will go away $100 \% . .$. so that's the expectation I do try to dampen down, because it's not realistic"'»c [24]. This is line with the findings of Lattig et al. [16] They reported that patients consistently had higher expectations than their spine surgeon regarding back pain and functioning (i.e., activities at work, household activities, and sports).

From the studies that measured post-operative fulfillment of expectations, some studies reported (at least 
Table 3 Synthesized finding 1: The majority of patients expected improvement on several domains after treatment, but these expectations were often overly optimistic

Findings
Patients undergoing spinal surgery
Patients planned for spinal surgery had much more optimistic expectations than their
surgeons about their likely pain and activity level 3 months postoperative [16]. [U]
Patients undergoing spinal surgery had high expectations of the treatment
outcomes [17]. [C]
Patients who had decompression surgery without fusion had overly optimistic
expectations of the outcomes of surgery [22]. [U]
Most patients scheduled for surgical decompression for sciatica expected complete
recovery, including resolution of their back pain [24]. [U]
Patients undergoing lumbar disc herniation surgery had high expectations [25]. [U]
Patients who had elective lumbar surgery had fairly high expectations of their
postoperative QOL [6]. [U]
Patients who had undergone spinal fusion had overly optimistic expectations about
recovery [28]. [U]

\section{Patients receiving advanced cancer care}

A substantial proportion of patients with metastatic cancer had the inaccurate expectation that cure after immunotherapy was likely [31]. [U]

A large proportion of the patients with incurable lung cancer had inaccurate beliefs about the likelihood of cure from radiotherapy [32]. [U]

About half of the patients with metastatic disease understood that their treatment was not curable, especially in patients without known brain metastases [33]. [U]

Many patients with metastatic disease believed that radiation treatment could prolong their lives [33]. [U]

Less than half of the patients with advanced cancer understood that their treatment was non-curative [35]. [U]

Many patients with recurrent ovarian cancer thought that chemotherapy would have a moderate to high chance of curing their disease [36]. [U]

After consultation with their radiation oncologist, about one-quarter of the patients undergoing palliative radiotherapy persist to believe their cancer is curable [39]. [U]

After consultation with their radiation oncologist, about half of the patients undergoing palliative radiotherapy persist to believe that treatment will prolong their life [39]. [U]

Almost half of the patients with lung cancer undergoing palliative chemotherapy were convinced that chemotherapy will cure them [40]. [U]

Some patients receiving palliative radiotherapy still expect/hope that their tumor will go away [42]. [C]

A substantial proportion of patients with advanced cancer had the inaccurate expectation that cure after chemotherapy was likely [43]. [U]

Many patients with metastatic lung or colorectal cancer who had opted to receive chemotherapy had inaccurate expectations about the curative potential of chemotherapy [44]. [U]

\section{Patients undergoing spinal surgery}

Patients' expectations exceeded the actual outcome.

Expectations from patients who had undergone spinal surgery frequently exceeded the actual outcome [15]. [U]

In more than half of the patients who had undergoing lumbar spinal surgery, expectations were not fulfilled [21]. [U]

Patients who had undergone spinal surgery expected better outcomes than they achieved [23]. [U]

Prepoperative expectations were higher than their fulfilled postoperative expectations in patients who had undergone lumbar surgery [6]. [U]

Most expectations of patients who had undergone spinal fusion regarding the postoperative period were not fulfilled [28]. [U]

In patients who had undergone spinal surgery, outcomes were not better than expected

Categories

Patients had overly optimistic expectations

about treatment outcomes. 
Table 3 Synthesized finding 1: The majority of patients expected improvement on several domains after treatment, but these expectations were often overly optimistic (Continued)

\begin{tabular}{ll}
\hline Findings & Categories
\end{tabular}

and even worse. However, some expectations were met [30]. [U]

\section{Patients receiving advanced cancer care}

Patients with advanced cancer expected that they would benefit more from chemotherapy than they actually did [37]. [U]

Expected benefits from chemotherapy were higher than experienced benefits in patients who were treated with chemotherapy [41]. [U]

\section{Patients undergoing spinal surgery}

Patients undergoing cervical spine surgery had diverse expectations that encompass improvement after surgery [18]. [U]

Patients undergoing lumbar spine surgery expected that they would improve on many areas [19]. [U]

Patients expected (much) improvement after decompression surgery [22]. [U]

Patients planned for spinal surgery had optimistic expectations regarding post-treatment outcomes [26]. [U]

Most patients undergoing spinal surgery expected that surgery will be successful and will relieve their symptoms [27]. [U]

Patients undergoing spinal surgery had high expectations for relief of leg pain, improvement in sleep and return to household and recreational activities, and lower expectation for return to work-related activities [29]. [U]

Preoperatively, patients undergoing spinal surgery expected significant improvements in postoperative outcomes [30].[U]

\section{Patients receiving advanced cancer care}

Patients with recurrent ovarian cancer have positive expectations of chemotherapy [36]. [C]

Almost all patients with advanced cancer expected improvement from chemotherapy [41]. [U]

\section{Patients undergoing spinal surgery}

Expectations were reached in approximately half of the patients who had undergone spinal surgery [17]. [U]

Almost all patients who had lumbar surgery had at least some of their expectations fulfilled [20]. [U]

Almost all patients who had cervical surgery had at least some of their expectations fulfilled [20]. [U]

Expectations were met in most of the patients who had undergone spinal surgery [27]. [U]

Patient expectations regarding post-treatment outcomes for spinal surgery were met in the majority of patients [29]. [U]

\section{Patients undergoing spinal surgery}

In patients who had lumbar surgery, the amount of improvement expected in pain was the expectation most often fulfilled [20]. [U]

In patients who had lumbar surgery, return to work was the expectation least often fulfilled [20]. [U]

In patients who had cervical surgery, the expected improvement in the ability to perform daily activities was the expectation most often fulfilled [20]. [U]

In patients who had cervical surgery, return to work was the expectation least often fulfilled [20]. [U]

Expectations regarding pain were most often fulfilled, while expectations regarding return to work and ability to work the least often fulfilled in patients who were planned for spinal surgery [26]. [U]

C credible, $U$ Unequivocal

In the majority of the patients, some of the expectations were met.

Fulfillment of expectations differed between expectations. 
some) expectations regarding post-operative outcomes were met in the majority of the patients who had undergone spinal surgery $[17,20,27,29]$. However, other studies reported that expectations were not fulfilled and exceeded actual outcomes [6, 15, 21, 23, 28, 30]. Van der Horst et al. reported that "more than half of the participants $(n=7)$ had expected recovery to be easier or at least a more upwards trajectory instead of the struggle they experienced in reality." [28] Likewise, a patient in the study of Accardi-Ravid et al. said: "The only thing that really surprised me-I was completely disabled as far as being mobile. And maybe they didn't explain that to me, to my full benefit, that I would be completely disabled as far as walking and stuff like that. I thought I'd have more ambulatory ability... I had to really work on that as far as learning how to walk again and I had to use a walker and practice." [15] Mancuso et al. studied expectations of several domains in patients who had lumbar or cervical spine surgery, and concluded that expectations regarding pain were most often met, while return to work the least often [20].

Studies regarding expectations of patients with advanced cancer reported that patients expected improvement from chemotherapy (e.g., symptom relief and feeling better) [36, 41], but these expectations were overly optimistic [37, 41]. Most of these studies reported that patients with advanced cancer believe that their cancer is still curable and that the planned treatment (radiotherapy, chemotherapy or immunotherapy) is likely to cure their disease. For example, Sze et al. illustrated: "Patients were able to acknowledge the terminal nature of their illness and still remain hopeful for a cure or remission. One lady said, "I'll be around for another ten years. This brain tumor is going to be cured. It's going to be shrunk to nothin". However, when asked regarding the prognosis of her disease she said, "Well it's not curable disease". At times, there appeared to be a tension between a patient's hope for cure and a suppressed realization of their true prognosis." [42]

\section{Finding 2: patient counseling is important for patients' understanding of disease and treatment}

Information provided by spine surgeons was important for patients scheduled for spinal surgery (Table 4). Accardi-Ravid et al. reported that some patients who had undergone spinal surgery indicated that their surgeon managed their preoperative expectations by negotiating the treatment objectives and explaining the expected results to the patient [15]. Rehman et al. identified different methods for surgeons to improve patient understanding, e.g., stimulating further deliberation or calling the patient the night before the surgery [24]. However, other patients did not feel adequately prepared for surgery and "patients often mentioned vague, positive qualifiers in response to what they expected from surgery", for example: "To be honest, I thought I would go into-at least I felt like I was led to believe that-I would have the surgery, the pain would be relieved, and my neck would be stable and I could go on with my life." and "I thought that I would just hang out in the hospital bed, take my drugs, be on my phone, and just watch $T V$." [15] Lattig et al. found patients and spine surgeons to have a different understanding of the terms associated with spinal disease, which may have resulted in overly optimistic patient expectations [16]. Rehman et al. concluded that providing an excessive amount of information was a barrier in disease and treatment understanding. Patients forgot information given by the spine surgeon or they were not able to process the information [24]. For example one spine surgeon expressed: "In spite of explaining everything with the help of patient images and/or models, I am surprised how little they actually take home."

When asking patients with advanced cancer whether they understood the incurable nature of their disease, many studies reported that the majority of patients understood the treatment goals, i.e., palliation, symptom relief, and/or improvement of quality of life [33, 39, 40]. According to Sze et al., patients with advanced cancer had varying information needs for future decision making, e.g., about prognosis: “... while some patients want more information, others have less need for knowledge to facilitate their decision making, preferring instead to defer to the knowledge of their doctors." [42]

\section{Finding 3: patient expectations were influenced by various factors, including age, health condition and socioeconomic status}

Multiple demographic, psychological and clinical characteristics were associated with patient expectations regarding treatment outcomes (Table 5). Younger patients undergoing spinal surgery who were physically and functionally more impaired had higher expectations (e.g., expected complete improvement) [18, 19]. One study found that better general health before surgery was associated with higher preoperative expectations [29], while another study found no association [27].

In patients with advanced cancer, one study found that older patients had more realistic expectations of cure [31]. In contrast, Chen et al. concluded that older patients were more likely to believe that they could be cured with palliative treatment [32]. A higher income was associated with more accurate and more optimistic expectations [31, 40]. Chow et al. reported that disease 
Table 4 Synthesized finding 2: Patient counseling is important for patients' understanding of disease and treatment

\section{Findings}

Patients undergoing spinal surgery

Some patients who had undergone spinal surgery did not feel adequately prepared for surgery [15]. [U]

Patients who had undergone spinal surgery could often not describe preoperatively what they expect from the recovery process [15]. [C]

The amount of information presented during the consultation with patients scheduled for surgical decompression for sciatica was excessive, and therefore, patients forget information that was given by the surgeon or don't get the message [24]. [U]

\section{Patients receiving advanced cancer care}

Most patients referred for palliative radiotherapy reported that they were dissatisfied with the information from the referring physician [33]. [U]

\section{Patients receiving advanced cancer care}

About half of the patients with metastatic disease understood that palliative radiotherapy could relieve their symptoms [33]. [U]

Advanced cancer patients with a low life expectancy had a clearer understanding of the treatment goal [35]. [U]

After discussing with the radiation team, patients undergoing palliative radiotherapy have a better understanding of their cancer, intent of radiation treatment and are less worried about receiving treatment [39]. [U]

The majority of patients with incurable lung cancer stated that chemotherapy aims to alleviate symptoms and improve quality of life [40]. [U]

The majority of patients with lung cancer undergoing palliative chemotherapy stated that chemotherapy will prolong their life [40]. [U]

The majority of patients with lung cancer declared that they are knowledgeable about palliative chemotherapy [40].[U]

\section{Patients undergoing spinal surgery}

Additional information was sought by patients who were planned for spinal surgery from external sources [24]. [U]

\section{Patients receiving advanced cancer care}

Patients receiving palliative radiotherapy (and their caregivers) have different information needs [42]. [U]

\section{Patients undergoing spinal surgery}

Some patients who had undergone spinal surgery indicated that their surgeon prepared them preoperatively on what to expect [15]. [C]

Patients and spine surgeons seem to have a different understanding of the terms associated with spinal diseases and hence different expectations, resulting in discrepancies between patient and surgeon expectations [16]. [C]

When the spine surgeon is more experienced, the surgeon has lower expectations than their patient [16]. [C]

There is variation in methods from spine surgeon to improve patients' understanding [24]. [U]

\section{Patients receiving advanced cancer care}

Almost half of initial palliative care conversations with patients with metastatic cancer included at least one statement regarding expectations for the patient's length of life [38]. [U]

$C$ credible, $U$ Unequivocal

characteristics (e.g., Karnofsky performance status, site of primary cancer and metastases, and symptom distress) did not affect expectations of cure [33]. However, pain at diagnosis influenced expectations regarding posttreatment pain reduction [34].

\section{Categories}

Pre-treatment consultation and patients' understanding and expectations

Patients declared that they understood the treatment goal.

Information needs of patients.

Patients' disease and treatment understanding and expectations were dependent on their surgeon.

\section{Discussion}

This systematic review synthesized findings of expectations regarding treatment outcomes from patients undergoing spinal surgery for non-cancer related conditions and from patients with advanced cancer. Assuming 
Table 5 Synthesized finding 3: Patient expectations were influenced by various factors, including age, health condition and socioeconomic status

Findings
Patients undergoing spinal surgery
Younger and more disabled patients who were scheduled for cervical spine surgery
expected complete improvement in more areas [18]. [U]
Multiple demographic, psychological, and clinical characteristics affect expectations of
patients scheduled for lumbar spine surgery [19]. [U]
Level of optimism and expectations were not correlated in patients undergoing elective
lumbar surgery [6]. [U]
Patients undergoing spinal surgery with better general health but poorer physical health
reported higher expectations [29]. [U]
Categories

Patients receiving advanced cancer care

Patients with metastatic cancer with an older age, higher income, and lower rates of anxiety had more accurate expectations of cure from immunotherapy [31]. [U]

Patients with incurable lung cancer who have inaccurate beliefs about radiotherapy also have inaccurate beliefs about chemotherapy [32]. [U]

Especially patients with incurable lung cancer who were older, non-Caucasian, and who completed the survey by themselves (instead of by surrogates) were more likely to have inaccurate believes about radiotherapy [32]. [U]

Patients with bone metastases with more pain expected a greater magnitude of pain reduction after palliative radiotherapy [34]. [U]

Patients with lung cancer undergoing palliative chemotherapy and living in large towns and good economic status were more optimistic about the hope for cure, prolonging life and alleviation of symptoms [40]. [U]

Hope and knowledge direct patient expectations of palliative radiotherapy [42]. [U]

Especially non-Caucasian patients, patients with lung cancer, patients who received care outside an integrated health care network, and patients who reported higher scores for physician communication had more often inaccurate expectations [44]. [U]

\section{Patients undergoing spinal surgery}

Characteristics that were not related to expectations.

Functional health status was not related to expectations of surgery in patients undergoing spinal surgery [27]. [U]

\section{Patients receiving advanced cancer care}

Disease characteristics and complaints had no impact on perceptions of cure in patients with metastatic disease [33]. [U]

\section{Patients receiving advanced cancer care}

Family and expectations.

Caregivers wanted to maintain patients' hope/expectations for cure after palliative radiotherapy [42]. [C]

$C$ credible, $U$ Unequivocal

that patients with spinal metastases share characteristics with these two populations, we studied patient expectations of these populations and draw parallels with the metastatic spine population. Based on these synthesized findings, we can conclude that patients who undergo spinal surgery and patients with advanced cancer tend to have overly optimistic expectations regarding treatment outcomes including pain and symptom relief, lower functional disability, (complete) recovery and prognosis. Discussing expected pain and symptom relief, recovery and prognosis before treatment may improve understanding of prognosis and promote and manage realistic expectations, which, in turn, may lead to better perceived outcomes and satisfaction.

\section{Characteristics that influenced expectations.}

Two previous systematic reviews concluded that higher preoperative expectations in patients undergoing spinal surgery predict higher post-operative satisfaction, improved functional outcomes and pain relief, but findings were not consistent [45-47]. In these reviews, no distinction was made between realistic and overly optimistic expectations. It may be that realistic positive expectations of post-operative improvement are associated with positive outcomes, but that overly optimistic expectations are associated with less favorable outcomes. Studies in patients undergoing other orthopedic surgical procedures (e.g., lower limb joint replacement) showed that not fulfilling pre-operative expectations was a strong predictor for dissatisfaction after surgery $[48,49]$. Because patients with overly optimistic expectations are less satisfied with their 
post-operative health status, it is likely that they rate their quality of life lower. Saban et al. found that fulfillment of expectations was associated with higher quality of life [6].

Physicians have an important role in supporting patients to develop realistic expectations. Excessive amounts of information provided by physicians result in limited recall and diminished understanding of the disease and surgery [24]. In addition, discrepancies between patients and physicians in understanding of the medical terms associated with spinal disease might affect how patients appraise the information provided (e.g., what patients remember after consultation and which message they take home). This might result in overly optimistic expectations. Multiple studies in this review concluded that expectations of patients undergoing spinal surgery were too high and often not fulfilled after surgery. Given the impact of unfulfilled expectations on post-operative satisfaction and quality of life, it is important that physicians review patient expectations before surgery, and adjusts where needed.

Patients with advanced cancer often acknowledged that they understand the palliative treatment goal. Yet, most patients still expected that treatment will cure their disease. This contradiction raises the question as whether patients really understand the meaning and implication of palliation. Lay language and insuring that the patient understands the vocabulary used is critical. It is important that patients have realistic expectations of their prognosis because this will help them acknowledge their incurable disease status and engage in end-of-life planning discussions [50]. In addition, patients with unrealistic expectations of treatment outcomes may accept invasive and toxic treatments, which they would not have accepted when they had developed more realistic expectations. Discussing prognosis with the patient may help patients to develop a better understanding of the incurable nature of their disease [51].

Heterogeneity exists in characteristics influencing expectations. For example, physically more disabled patients were inclined to have more unrealistic expectations. Therefore, an individualized approach is essential in which the physician explores individual patient expectations and when unrealistic, subsequently tries to influence these expectations.

The incurable nature of metastatic spine disease may affect patient expectations after treatment which set them apart from the degenerative spine population. Although patients with metastatic spine disease often receive advanced cancer care, these patients may face unique challenges such as neurologic deficit and as such, their expectations may differ from those patients without spinal metastases. The treatment approach of patients with spinal metastases is often multidisciplinary (e.g., a medical or radiation oncologist, a spine surgeon, an oncology nurse), which may hamper consistent information disclosure. Therefore, more research is needed to gain insight into expectations of treatment outcomes in patients with spinal metastases and the best methods to instill appropriate or realistic expectations.

\section{Conclusions}

Patients tend to have overly optimistic expectations regarding pain and symptom relief, recovery and prognosis. Pretreatment discussion about the expected pain and symptom relief, recovery and prognosis may improve understanding of prognosis, and promote and manage realistic expectations, which, in turn, may lead to higher satisfaction with the treatment outcome and hence a higher quality of life.

\section{Supplementary Information}

The online version contains supplementary material available at https://doi. org/10.1186/s12885-020-07683-7.

Additional file 1. Search strategies

\section{Abbreviations}

CASP: Critical Appraisal Skills Programme; ENTREQ: Enhancing Transparency in Reporting the Synthesis of Qualitative Research; HRQOL: Health related quality of life; MESCC: Metastatic epidural spinal cord compression

\section{Acknowledgements}

The authors want to thank the P.H. Wiersma of the Utrecht University for the help with developing the search strategy.

\section{Authors' contributions}

AV and RG were responsible for the design and concept of the study. AV and RG developed the search strategy and searched for the studies. AV, DO and RG selected the studies. DO and RG conducted the data extraction and data analysis. RG drafted the manuscript and DO, HW, JV, RCM, CF, HV and AV read and critically revised the paper. All authors approved the final manuscript.

\section{Funding}

This study was funded by AOSpine International. The funder had no role in study design, data collection and analysis, interpretation of data, and preparation of the manuscript.

\section{Availability of data and materials}

Data sharing is not applicable to this article as no datasets were generated or analysed during the current study.

Ethics approval and consent to participate Not applicable.

\section{Consent for publication}

Not applicable.

\section{Competing interests}

The authors have no conflicts of interest to disclose that could have influenced the work reported in this article. Dr. CG Fisher reports consulting fees and royalties from Medtronic, consulting fees from Nuvasive and receives fellowship support paid by Medtronic and AO Spine, not relevant for this article. Dr. JJ Verlaan received an educational grant from DePuy Synthes, and is co-founder of, and holds stocks in SentryX, not relevant for this article. Dr. AL Versteeg received consultancy and travelling support from AO Spine, not relevant for this article. 


\section{Author details}

'Division of Imaging and Cancer, University Medical Center Utrecht, University of Utrecht, Heideberglaan 100, 3584, CG, Utrecht, the Netherlands. ${ }^{2}$ Department of Orthopaedic Surgery, University Medical Center Utrecht, University of Utrecht, Utrecht, the Netherlands. ${ }^{3}$ Department of Corporate Communications, University Medical Center Utrecht, Utrecht, The Netherlands. ${ }^{4}$ Department of Orthopaedics, Division of Spine, Vancouver General Hospital/University of British Columbia, Vancouver, British Columbia, Canada.

Received: 10 July 2020 Accepted: 23 November 2020 Published online: 09 December 2020

\section{References}

1. Cole JS, Patchell RA. Metastatic epidural spinal cord compression. Lancet Neurol. 2008;7(5):459-66

2. Coleman RE. Metastatic bone disease: clinical features, pathophysiology and treatment strategies. Cancer Treat Rev. 2001;27(3):165-76.

3. Macedo F, Ladeira K, Pinho F, Saraiva N, Bonito N, Pinto L, Goncalves F. Bone metastases: an overview. Oncol Rev. 2017:11(1):321.

4. Spratt DE, Beeler WH, de Moraes FY, Rhines LD, Gemmete JJ, Chaudhary N, Shultz DB, Smith SR, Berlin A, Dahele M, et al. An integrated multidisciplinary algorithm for the management of spinal metastases: an international spine oncology consortium report. Lancet Oncol. 2017;18(12): e720-30.

5. The World Health Organization Quality of Life assessment (WHOQOL): position paper from the World Health Organization. Soc Sci Med. 1995; 41(10):1403-9.

6. Saban KL, Penckofer SM. Patient expectations of quality of life following lumbar spinal surgery. J Neurosci Nurs. 2007;39(3):180-9.

7. Conner-Spady BL, Bohm E, Loucks L, Dunbar MJ, Marshall DA, Noseworthy TW. Patient expectations and satisfaction 6 and 12 months following total hip and knee replacement. Qual Life Res. 2020;29(3):705-19.

8. Thompson AG, Sunol R. Expectations as determinants of patient satisfaction: concepts, theory and evidence. Int J Qual Health Care. 1995:7(2):127-41.

9. Witiw CD, Mansouri A, Mathieu F, Nassiri F, Badhiwala JH, Fessler RG Exploring the expectation-actuality discrepancy: a systematic review of the impact of preoperative expectations on satisfaction and patient reported outcomes in spinal surgery. Neurosurg Rev. 2018;41(1):19-30.

10. Moher D, Liberati A, Tetzlaff J, Altman DG, Group P. Preferred reporting items for systematic reviews and meta-analyses: the PRISMA statement. PLOS Med. 2009;6(7):e1000097.

11. CASP Qualitative Checklist.: https://casp-uk.net/casp-tools-checklists/; 2019.

12. CASP Cohort Study Checklist: https://casp-uk.net/casp-tools-checklists/; 2019

13. Lockwood C, Oh EG. Systematic reviews: guidelines, tools and checklists for authors. Nurs Health Sci. 2017;19(3):273-7.

14. Lockwood CRN, Munn Z, Porritt K: Qualitative research synthesis. Int J Evid Based Healthc 2015, 13(3):179-187.

15. Accardi-Ravid M, Eaton L, Meins A, Godfrey D, Gordon D, Lesnik I, Doorenbos A. A qualitative descriptive Study of patient experiences of pain before and after spine surgery. Pain Med. 2020;21(3):604-12.

16. Lattig F, Fekete TF, O'Riordan D, Kleinstuck FS, Jeszenszky D, Porchet F, Mutter U, Mannion AF. A comparison of patient and surgeon preoperative expectations of spinal surgery. Spine (Phila Pa 1976). 2013;38(12):1040-8.

17. Licina P, Johnston M, Ewing L, Pearcy M. Patient expectations, outcomes and satisfaction: related, relevant or redundant? Evid Based Spine Care J. 2012:3(4):13-9.

18. Mancuso CA, Duculan R, Stal M, Girardi FP. Patients' expectations of cervical spine surgery. Spine (Phila Pa 1976). 2014;39(14):1157-62.

19. Mancuso CA, Duculan R, Stal M, Girardi FP. Patients' expectations of lumbar spine surgery. Eur Spine J. 2015:24(11):2362-9.

20. Mancuso CA, Duculan R, Cammisa FP, Sama AA, Hughes AP, Lebl DR, Girardi FP. Fulfillment of patients' expectations of lumbar and cervical spine surgery. Spine J. 2016;16(10):1167-74.

21. Mancuso CA, Reid MC, Duculan R, Girardi FP. Improvement in pain after lumbar spine surgery: the role of preoperative expectations of pain relief. Clin J Pain. 2017;33(2):93-8

22. Mannion AF, Junge A, Elfering A, Dvorak J, Porchet F, Grob D. Great expectations: really the novel predictor of outcome after spinal surgery? Spine (Phila Pa 1976). 2009;34(15):1590-9.
23. McGregor AH, Dore CJ, Morris TP. An exploration of patients' expectation of and satisfaction with surgical outcome. Eur Spine J. 2013;22(12):2836-44.

24. Rehman Y, Syed M, Wiercioch W, Rehman N, Drew B, Cenic A, Reddy K, Murty N, Kucher E, Dunlop B, et al. Discrepancies between patient and surgeon expectations of surgery for sciatica: a challenge for informed decision making? Spine (Phila Pa 1976). 2019;44(10):740-6.

25. Ronnberg K, Lind B, Zoega B, Halldin K, Gellerstedt M, Brisby H. Patients' satisfaction with provided care/information and expectations on clinical outcome after lumbar disc herniation surgery. Spine (Phila Pa 1976). 2007; 32(2):256-61.

26. Soroceanu A, Ching A, Abdu W, McGuire K. Relationship between preoperative expectations, satisfaction, and functional outcomes in patients undergoing lumbar and cervical spine surgery: a multicenter study. Spine (Phila Pa 1976). 2012;37(2):E103-8.

27. Toyone T, Tanaka T, Kato D, Kaneyama R, Otsuka M. Patients' expectations and satisfaction in lumbar spine surgery. Spine (Phila Pa 1976). 2005;30(23): 2689-94.

28. van der Horst AY, Trompetter HR, Pakvis DFM, Kelders SM, Schreurs KMG, Bohlmeijer ET. Between hope and fear: a qualitative study on perioperative experiences and coping of patients after lumbar fusion surgery. Int J Orthop Trauma Nurs. 2019:35:100707.

29. Yee A, Adjei N, Do J, Ford M, Finkelstein J. Do patient expectations of spinal surgery relate to functional outcome? Clin Orthop Relat Res. 2008;466(5): 1154-61.

30. Yoo JS, Patel DV, Mayo BC, Massel DH, Karmarkar SS, Lamoutte EH, Singh K. Postoperative satisfaction following lumbar spinal fusion surgery: patient expectation versus actuality. J Neurosurg Spine. 2019:1-7.

31. Bergerot CD, Bergerot PG, Philip EJ, Hsu JA, Dizman N, Vaishampayan U, Dorff T, Pal SK. Perception of cure among patients with metastatic genitourinary cancer initiating immunotherapy. J Immunother Cancer. 2019; 7(1):71.

32. Chen AB, Cronin A, Weeks JC, Chrischilles EA, Malin J, Hayman JA, Schrag D. Expectations about the effectiveness of radiation therapy among patients with incurable lung cancer. J Clin Oncol. 2013;31(21):2730-5.

33. Chow E, Andersson L, Wong R, Vachon M, Hruby G, Franssen E, Fung KW, Harth T, Pach B, Pope J, et al. Patients with advanced cancer: a survey of the understanding of their illness and expectations from palliative radiotherapy for symptomatic metastases. Clin Oncol (R Coll Radiol). 2001; 13(3):204-8.

34. Chow E, Chiu H, Doyle M, Hruby G, Holden L, Barnes EA, Tsao M, Mallia G, Harris K, Danjoux C. Patient expectation of the partial response and response shift in pain score. Support Cancer Ther. 2007:4(2):110-8.

35. Craft PS, Burns CM, Smith WT, Broom DH. Knowledge of treatment intent among patients with advanced cancer: a longitudinal study. Eur J Cancer Care (Engl). 2005;14(5):417-25.

36. Doyle C, Crump M, Pintilie M, Oza AM. Does palliative chemotherapy palliate? Evaluation of expectations, outcomes, and costs in women receiving chemotherapy for advanced ovarian cancer. J Clin Oncol. 2001; 19(5):1266-74.

37. Friedlander ML, Stockler M, O'Connell R, Voysey M, Oza A, Gillies K, Donovan $H$, Martyn J, Sjoquist K, Butow P, et al. Symptom burden and outcomes of patients with platinum resistant/refractory recurrent ovarian cancer: a reality check: results of stage 1 of the gynecologic cancer intergroup symptom benefit study. Int J Gynecol Cancer. 2014;24(5):857-64.

38. Gramling R, Fiscella K, Xing G, Hoerger M, Duberstein P, Plumb S, Mohile S, Fenton JJ, Tancredi DJ, Kravitz RL, et al. Determinants of patient-oncologist prognostic discordance in advanced Cancer. JAMA Oncol. 2016;2(11):1421-6.

39. Mitera G, Zhang L, Sahgal A, Barnes E, Tsao M, Danjoux C, Holden L, Chow E. A survey of expectations and understanding of palliative radiotherapy from patients with advanced cancer. Clin Oncol (R Coll Radiol). 2012;24(2): 134-8.

40. Nowicki A, Wozniak K, Krajnik M. Understanding the purpose of treatment and expectations in patients with inoperable lung cancer treated with palliative chemotherapy. Contemp Oncol (Pozn). 2015;19(4):333-7.

41. Sjoquist KM, Friedlander ML, O'Connell RL, Voysey M, King MT, Stockler MR, Oza AM, Gillies K, Martyn JK, Butow PN. Hope, quality of life, and benefit from treatment in women having chemotherapy for platinum-resistant/ refractory recurrent ovarian cancer: the gynecologic cancer intergroup symptom benefit study. Oncologist. 2013;18(11):1221-8.

42. Sze J, Marisette S, Williams D, Nyhof-Young J, Crooks D, Husain A, Bezjak A, Wong RK. Decision making in palliative radiation therapy: reframing hope in 
caregivers and patients with brain metastases. Support Care Cancer. 2006; 14(10):1055-63.

43. Visser S, de Mol M, Cheung K, van Toor JJ, van Walree NC, Stricker BH, Den Oudsten BL, Aerts J. Treatment satisfaction of patients with advanced nonsmall-cell lung Cancer receiving platinum-based chemotherapy: results from a prospective Cohort Study (PERSONAL). Clin Lung Cancer. 2018;19(4):e503-16.

44. Weeks JC, Catalano PJ, Cronin A, Finkelman MD, Mack JW, Keating NL, Schrag D. Patients' expectations about effects of chemotherapy for advanced cancer. N Engl J Med. 2012;367(17):1616-25.

45. Ellis DJ, Mallozzi SS, Mathews JE, Moss IL, Ouellet JA, Jarzem P, Weber MH. The relationship between preoperative expectations and the short-term postoperative satisfaction and functional outcome in lumbar spine surgery: a systematic review. Global Spine J. 2015;5(5):436-52

46. Menendez JY, Omar NB, Chagoya G, Tabibian BE, Elsayed GA, Walters BC, Guthrie BL, Hadley MN. Patient satisfaction in spine surgery: a systematic review of the literature. Asian Spine J. 2019;13(6):1047-57.

47. Swarup I, Henn CM, Gulotta LV, Henn RF 3rd. Patient expectations and satisfaction in orthopaedic surgery: a review of the literature. J Clin Orthop Trauma. 2019;10(4):755-60.

48. Bourne RB, Chesworth BM, Davis AM, Mahomed NN, Charron KD. Patient satisfaction after total knee arthroplasty: who is satisfied and who is not? Clin Orthop Relat Res. 2010:468(1):57-63.

49. Hamilton DF, Lane JV, Gaston P, Patton JT, Macdonald D, Simpson AH, Howie CR. What determines patient satisfaction with surgery? A prospective cohort study of 4709 patients following total joint replacement. BMJ Open. 2013;3(4):e002525.

50. Lambden J, Zhang B, Friedlander R, Prigerson HG. Accuracy of Oncologists life-expectancy estimates recalled by their advanced Cancer patients: correlates and outcomes. J Palliat Med. 2016;19(12):1296-303.

51. Epstein AS, Prigerson HG, O'Reilly EM, Maciejewski PK. Discussions of life expectancy and changes in illness understanding in patients with advanced Cancer. J Clin Oncol. 2016;34(20):2398-403.

\section{Publisher's Note}

Springer Nature remains neutral with regard to jurisdictional claims in published maps and institutional affiliations.

Ready to submit your research? Choose BMC and benefit from:

- fast, convenient online submission

- thorough peer review by experienced researchers in your field

- rapid publication on acceptance

- support for research data, including large and complex data types

- gold Open Access which fosters wider collaboration and increased citations

- maximum visibility for your research: over $100 \mathrm{M}$ website views per year

At $\mathrm{BMC}$, research is always in progress.

Learn more biomedcentral.com/submissions 